\title{
Cambios en las concentraciones de retinol, hemoglobina y ferritina en niños palúdicos colombianos
}

\author{
Rosa Magdalena Uscátegui ${ }^{1}$, Adriana M. Correa², Jaime Carmona-Fonseca ${ }^{2}$ \\ ${ }^{1}$ Grupo de Investigación en Alimentación y Nutrición Humana, Escuela de Nutrición y Dietética, \\ Universidad de Antioquia, Medellín, Colombia \\ ${ }^{2}$ Grupo Salud y Comunidad, Facultad de Medicina, Universidad de Antioquia, Medellín, Colombia
}

Introducción. La malaria, la anemia y las parasitosis coexisten en niños colombianos. Se desconoce el efecto del suplemento de retinol y de los antiparasitarios sobre estos problemas de salud y la evolución de la hemoglobina, el retinol, la ferritina y la proteína $C$ reactiva, en niños palúdicos.

Objetivo. Comparar el efecto de la intervención simultánea con antipalúdicos, antiparasitarios y un suplemento de retinol sobre la evolución de la hemoglobina, la ferritina, el retinol y la proteína $C$ reactiva, en niños palúdicos.

Materiales y métodos. Estudio experimental no ciego de 93 niños palúdicos de 4 a 10 años, con asignación aleatoria a uno de los siguientes grupos: uno recibió antipalúdico y suplemento de retinol (grupo MA); otro, antipalúdico, suplemento de retinol y antiparasitarios (grupo MAP); otro, antipalúdico y antiparasitarios (grupo MP) y otro, sólo antipalúdico (grupo M); se siguieron 30 días, se evaluaron los cambios de hemoglobina, ferritina, retinol y proteína $C$ reactiva, 8 y 30 días después.

Resultados. En el total de los niños, el día del ingreso los promedios de las determinaciones fueron: hemoglobina, $10,3 \pm 1,6 \mathrm{~g} / \mathrm{dl}$; retinol, $19,1 \pm 6,0 \mu \mathrm{g} / \mathrm{dl}$; proteína C reactiva, $75 \pm 63 \mathrm{mg} / \mathrm{L}, \mathrm{y}$ ferritina, $213 \pm 203 \mu \mathrm{g} / \mathrm{L}$. Al día 30 , las concentraciones de hemoglobina y retinol aumentaron en $1,4 \pm 1,4 \mathrm{~g} / \mathrm{dl}$ y $11,5 \pm 8,1 \mu \mathrm{g} / \mathrm{dl}$, respectivamente, mientras que las concentraciones de proteína $\mathrm{C}$ reactiva y ferritina disminuyeron en $66 \pm 60 \mathrm{mg} / \mathrm{L}$ y $184 \pm 203 \mu \mathrm{g} / \mathrm{L}$, respectivamente, sin diferencias estadísticamente significativas entre los grupos de tratamiento. Los cambios el día 8 sólo fueron diferentes por grupo para la hemoglobina, que incrementó en el grupo MAP, diferente a los otros grupos.

Conclusión. El día 30, la hemoglobina y el retinol aumentaron, y la proteína C reactiva y la ferritina disminuyeron. El suplemento de retinol y antiparasitarios simultáneos previno la reducción de hemoglobina al día 8, sin afectar los cambios en otras variables.

Palabras clave: malaria, vitamina A, anemia, niño, Colombia.

Changes in retinol, hemoglobin and ferritin concentrations in Colombian children with malaria

Introduction. Malaria, anemia and intestinal parasitism can co-exist in certain populations of Colombian children. The effects of retinol supplementation and anti-intestinal parasite treatment in children with malaria is unknown. Changes after this treatment of with respect to hemoglobin, retinol, ferritin and $\mathrm{C}$ reactive protein levels have not been previously monitored.

Objective. The effect of simultaneous intervention with antimalarial, retinol supplementation and anti-intestinal parasites treatment will be monitored by examining levels of hemoglobin, ferritin, retinol and $C$ reactive protein in children with malaria.

Materials and methods. A non-blind experimental study was conducted in 93 children with malaria, aged 4-10 years. Each was randomly allocated to one of the following groups: (1) treatment with antimalarial and retinol supplement (Group MA); (2) treatment with antimalarialretinol supplement and anti-parasitic drug (Group MAP); (3) treatment with antimalarial and antiparasitic drug (Group MP), and (4) treatment only with antimalarials (Group M). The groups were 
observed for 30 days, with haemoglobin, ferritin, retinol and $\mathrm{C}$ reactive protein evaluated on days 0,8 and 30 after treatment.

Results. Mean values for the children at day 0 were as follows: hemoglobin $10.3 \pm 1.6 \mathrm{~g} / \mathrm{dL}$, retinol $19.1 \pm 6.0 \mu \mathrm{g} / \mathrm{dL}$, C reactive protein $75 \pm 63 \mathrm{mg} / \mathrm{L}$ and ferritin $213 \pm 203 \mu \mathrm{g} / \mathrm{L}$. On day 30 after treatment, hemoglobin and plasma retinol concentrations increased in $1.4 \pm 1.4 \mathrm{~g} / \mathrm{dL}$ and $11.5 \pm 8.1 \mu \mathrm{g} / \mathrm{dL}$, whereas the $\mathrm{C}$ reactive protein and ferritin concentrations decreased to $66 \pm 60$ $\mathrm{mg} / \mathrm{L}$, and $184 \pm 203 \mu \mathrm{g} / \mathrm{L}$, respectively. No statistically significant differences appeared among the groups. On day 8, significant differences between the groups were observed in hemoglobin concentrations Group MAP was higher when compared to other groups.

Conclusion. On day 30 , hemoglobin and retinol were high, whereas $\mathrm{C}$ reactive protein was low. Simultaneous administration of a retinol supplement and anti-parasite treatment prevented hemoglobin reduction observed on day 8 without changes in other variables.

Key words: malaria, vitamin A, anemia, child, Colombia.

El paludismo, la anemia y las parasitosis intestinales son problemas de salud pública en la población colombiana menor de 15 años (1-3). En 2006, Antioquia reportó 41.012 casos de malaria, de los cuales, 48,4\% ocurrieron en la región de Urabá (3.353,5 casos/100.000 habitantes) y 42,3\% en la del Bajo Cauca (7.915,4 casos/100.000 habitantes) (1); según estudios previos, $29 \%$ de esos casos corresponden a la población de 5 a14 años $(4,5)$.

El retinol plasmático, la ferritina y la hemoglobina son biomarcadores del estado nutricional de la vitamina $A$ y del hierro. El retinol es indicador de los depósitos de vitamina $A(6)$, la hemoglobina se usa para evaluar la anemia y la ferritina es indicador de los depósitos de hierro (7). Estas variables se afectan por la respuesta de fase aguda ante una infección: el retinol disminuye y la ferritina aumenta (8).

La malaria produce un prominente estado inflamatorio, indicado por las altas concentraciones de proteína C reactiva (9), acompañado de valores de retinol inferiores a $20 \mu \mathrm{g} / \mathrm{dl}(14 \mu \mathrm{g} /$ $\mathrm{dl})$, que se incrementan después del tratamiento antipalúdico, (10) y anemia con reportes muy variables entre la población colombiana que van desde $30 \%$ hasta $50 \%(11,12)$.

\footnotetext{
Correspondencia:

Rosa Magdalena Uscátegui, SIU, Universidad de Antioquia, Calle 62 № 52-59, laboratorio 413, apartado aéreo 1226, Medellín, Colombia.

Teléfono: (574) 219 6498; fax: (574) 219 6400, extensión 6669

rosauscategui@gmail.com
}

Recibido: 18/06/08; aceptado:09/02/09
En población no palúdica, los suplementos de vitamina A contribuyen a incrementar las concentraciones de hemoglobina (7) y, en la población con deficiencia subclínica de vitamina $A$, incrementan las concentraciones séricas de retinol. Por otra parte, varios estudios asocian la presencia de algunos helmintos intestinales con anemia y bajas concentraciones de retinol, que mejoran después de la administración de antihelmínticos $(13,14)$.

Los estudios sobre la evolución de la inflamación y de los biomarcadores del estado nutricional del hierro y de la vitamina $A$ en niños palúdicos, son escasos y fragmentarios, especialmente en la población colombiana. Tampoco se conoce el efecto de la administración simultánea con el tratamiento antipalúdico, de un suplemento de retinol y antiparasitarios intestinales, solos o combinados, sobre la evolución de la anemia, los bajos depósitos de retinol y la respuesta inflamatoria, en niños con malaria. El conocimiento de estos aspectos permite orientar la correcta evaluación del estado nutricional del hierro y de la vitamina $A$ en ese grupo de población y orienta las acciones más apropiadas para combatir la anemia en los niños de las zonas palúdicas.

Por esta razón, se realizó un estudio cuyo objetivo fue comparar el efecto sobre la evolución de la hemoglobina, la ferritina, el retinol y la proteína $\mathrm{C}$ reactiva, producido por la intervención simultánea con antipalúdicos, antiparasitarios (antihelmínticos más antiprotozoarios intestinales) y un suplemento oral de vitamina $A$, frente a otras 
opciones: sólo antipalúdicos; antipalúdicos más antiparasitarios, y antipalúdicos más vitamina $A$.

\section{Materiales y métodos}

\section{Clase de estudio}

Diseño experimental, no ciego, con asignación aleatoria al factor de exposición de 93 niños residentes en las zonas urbanas o rurales de los municipios de El Bagre y Turbo, que consultaron al puesto de diagnóstico de malaria de cada lugar por presentar síndrome febril y a quienes se les demostró, mediante examen de gota gruesa, que presentaban infección con Plasmodium falciparum o Plasmodium vivax, excluyendo las infecciones mixtas. Los niños debían cumplir con los siguientes criterios de inclusión: tener entre 4 y 10 años, presentar malaria confirmada por gota gruesa y que ésta no fuera complicada, según los criterios de la Organización Mundial de la Salud (OMS) (15), no presentar otra infección agregada ni enfermedad crónica, no haber consumido suplemento de vitamina $A$ durante los seis meses anteriores, ni haber presentado otro episodio de malaria durante el último mes.

Los niños se distribuyeron aleatoriamente entre cuatro grupos de tratamiento, así: uno recibió antipalúdico y suplemento de retinol (grupo $\mathrm{MA}$ ); otro, antipalúdico, suplemento de retinol y antiparasitarios (grupo MAP); otro, antipalúdico y antiparasitarios (grupo MP), y otro, sólo antipalúdico (grupo M).

\section{Unidad de análisis y diseño muestral}

Entre el 2000 y el 2003 en Turbo y en El Bagre, se presentaron 12.729 casos de paludismo en niños de 5 a 14 años, es decir, un promedio de 3.182 casos por año.

No hay antecedentes de estudios en los que se hayan administrado simultáneamente antiparasitarios intestinales y suplemento de retinol en niños con malaria. Sin embargo, con base en los resultados de investigaciones en niños palúdicos y no palúdicos, en quienes se evaluó la diferencia en los valores de hemoglobina atribuibles al uso de suplementos de vitamina $A$ o de antiparasitarios intestinales (16-19), se estimó que 26 niños por grupo serían suficientes para detectar $0,7 \mathrm{~g} / \mathrm{dl}$ de diferencia en la hemoglobina entre el grupo control y el que recibió las dos intervenciones, asumiendo una desviación estándar de $0,9 \mathrm{~g} / \mathrm{dl}$, un poder de $80 \%$ y una confianza de $95 \%$. En consecuencia, se decidió incluir en el estudio a 52 niños residentes en El Bagre y 52 en Turbo.

A cada uno de los participantes se le hizo una evaluación clínica general, antropométrica, bioquímica y examen coprológico, al ingresar al estudio, es decir, antes del tratamiento antipalúdico y en los días 8 y 30 después de éste. El diagnóstico de malaria se hizo con gota gruesa y examen de extendido de sangre periférica, según el procedimiento indicado por la OMS (20). El tratamiento antipalúdico se administró por vía oral, para $P$. vivax, cloroquina (tabletas de $150 \mathrm{mg}$ base), dosis total de $25 \mathrm{mg} /$ $\mathrm{kg}$ en tres días, y primaquina (tabletas de 15 $\mathrm{mg}$ ) en dosis única diaria de $0,50 \mathrm{mg} / \mathrm{kg}$ por 7 días, según los esquemas del Ministerio de la Protección Social de Colombia y la Dirección Seccional de Salud de Antioquia (medicamento y dosis según edad); se suministraron en forma supervisada por los investigadores. Para $P$. falciparum se dio amodiaquina (tabletas de 150 $\mathrm{mg}$ base), dosis total de $25 \mathrm{mg} / \mathrm{kg}$ en tres días; más sulfadoxina-pirimetamina (tabletas de 500 $\mathrm{mg}$ base de sulfadoxina/25 mg pirimetamina), en dosis única, $25 \mathrm{mg} / \mathrm{kg}$ de sulfadoxina y $1 \mathrm{mg} /$ $\mathrm{kg}$ de pirimetamina; más primaquina (tabletas $15 \mathrm{mg}$ ) dosis única de $0,75 \mathrm{mg} / \mathrm{kg}$ peso.

\section{Tratamiento antiparasitario y suplemento de vitamina $A$}

Se administraron por vía oral. Cada niño recibió una sola dosis de $400 \mathrm{mg}$ de albendazol en suspensión del Laboratorios MK, 500 mg desecnidazol en suspensión, de Genéricos Medícalex (Biogen $\AA^{8}$ ) y vitamina $A$ en forma de palmitato de retinol, 200.000 unidades internacionales (UI), provenientes de dos tabletas de Retiblan ${ }^{\circledR}$ (Procaps) de $100.000 \mathrm{UI}$ cada una.

\section{Proteína C reactiva}

Se midió en suero, mediante un estuche BioSystems (proteína $C$ reactiva) Látex $\AA$, por la técnica de turbidimetría (21). Se consideró que 
había inflamación cuando la concentración de proteína $\mathrm{C}$ reactiva era superior a $8 \mathrm{mg} / \mathrm{L}$.

\section{Retinol plasmático}

Se midió en el Instituto Nacional de Salud por cromatografía líquida de alta eficacia (high performance liquid chromatography, HPLC) en muestras de plasma protegidas de la luz (22), con un equipo marca Waters, mediante un sistema de inyección manual Reodyne 77251, un sistema de suministro de solventes 660E, un detector UV-VIS 400 y una columna C-18 Symmetry. Se hicieron dos cromatogramas por cada muestra evaluada. Se consideró que había deficiencia subclínica de vitamina $A$ cuando los valores plasmáticos estaban por debajo de $20 \mu \mathrm{g} / \mathrm{dl}$.

\section{Hemoleucograma}

Se hizo hemoleucograma tipo III-V en equipo automatizado, por el método de cianometahemoglobina recomendado por la OMS, en el Laboratorio Clínico del Hospital Francisco Valderrama de Turbo y en el Laboratorio Medicauca de El Bagre.Losvalores de referenciaparadiagnosticar anemia fueron los recomendados por la OMS según la concentración de hemoglobina, así: para los niños de 4 años, menos de $11,0 \mathrm{~g} /$ $\mathrm{dl}$, y para los de 5 a 10 años, menos de $12 \mathrm{~g} /$ dl (7). Los valores de hemoglobina no fueron corregidos por altitud, porque El Bagre y Turbo están situados a menos de 200 metros sobre el nivel del mar.

\section{Ferritina}

Se midió en suero, con el estuche Abbott AxSYM $^{\circledR}$ System 7A58-20 B7A583 56-4324/ R12, Abbott Laboratorios, USA. El ensayo AxSYM Ferritin se basa en la tecnología de inmunoanálisis enzimático de micropartículas (microparticle enzyme immunoassay, MEIA). Estas determinaciones y las de proteína C reactiva se hicieron en el Laboratorio Clínico de la IPS de la Universidad de Antioquia.

\section{Evaluación antropométrica}

Se obtuvieron el peso y la talla con equipos y técnicas de uso internacional. Los datos de la evaluación antropométrica fueron publicados en otro artículo (23).

\section{Examen coprológico}

El examen de las muestras fecales se hizo al inicio del estudio (día 0) y a los días 8 y 30, con muestra única. Se estableció la presencia de helmintos y protozoos por examen directo; cuando éste no mostró ningún parásito, se hizo examen por concentración con formol-éter (24). Sólo cuando la segunda evaluación era negativa, se declaró como tal a la muestra. En las muestras positivas para helmintos se hizo recuento en placa para conocer el número de huevos por gramo de materia fecal, según la técnica de Kato-Katz (24). Los exámenes fueron realizados por el Grupo Interdisciplinario para el Estudio de las Parasitosis Intestinales de la Facultad de Medicina de la Universidad de Antioquia.

\section{Análisis estadístico}

La comparación de las variables cuantitativas entre los grupos de tratamiento en la línea de base (día 0 ), se realizó mediante la prueba $\mathrm{H}$ de Kruskall y Wallis para muestras independientes y para las variables categóricas, como anemia, inflamación y deficiencia subclínica de vitamina A, la comparación entre los cuatro grupos de tratamiento, al inicio y al final del estudio, se realizó mediante la prueba de ji al cuadrado.

En cada grupo se calculó el cambio total, correspondiente a la diferencia entre el valor del día 30 y el de la línea de base, de cada una de las variables bioquímicas. Igualmente, se calculó el cambio parcial, restando el valor de la línea de base al de la segunda medición (día 8). La comparación del cambio total y del parcial entre los grupos de tratamiento, se realizó por la prueba de Kruskall y Wallis. En cada grupo y para cada variable, se compararon el cambio total y el parcial, mediante la prueba de Wilcoxon. Las diferencias entre el inicio y el final del estudio para las variables cuantitativas, se midieron con la prueba de Wilcoxon, y para las variables categóricas, mediante la prueba de control de cambios de McNemar.

Los análisis se realizaron por medio de los programas Prisma y el SPSS versión 15.0 y se aceptaron como significativos valores de $p<0,05$. 


\section{Consideraciones éticas}

El acudiente de cada niño recibió una explicación detallada del proyecto y firmó el consentimiento informado. El estudio fue avalado por el Comité de Ética del Centro de Investigaciones Médicas de la Universidad de Antioquia.

\section{Resultados}

Se captaron 97 niños durante el año 2005; cuatro niños se perdieron durante el seguimiento de un mes y, finalmente, quedaron 93 niños: 21 en el grupo con ambos tratamientos adicionales al antipalúdico (MAP), 23 en el grupo MP, 26 en el grupo MA y 23 en el grupo M. Se hizo examen coprológico en 85 niños, 20 del grupo MAP, 20 del MP, 23 del MA y 22 del M. De los 93 niños que permanecieron durante el seguimiento, se excluyeron del análisis de los datos bioquímicos del día 30, tres niños que presentaron gota gruesa positiva y cinco más a quienes no fue posible tomarles las muestras. Por consiguiente, quedaron 85 niños distribuidos en los diferentes grupos así: grupo MAP, 18; grupo MP, 21; grupo MA, 24, y grupo M, 22. Cabe anotar que únicamente a 44 niños se les determinó el retinol el día 8 y, de ellos, pertenecían 9 al grupo MAP, 9 al grupo M, 12 al grupo MP y 14 al MA.

El 65\% eran hombres, con similar distribución entre los cuatro grupos $(p=0,994)$. La edad promedio fue de 7,0 años, sin diferencias estadísticamente significativas entre los grupos $(p=0,944)$, los que también fueron similares en peso y talla. De los 93 casos de paludismo, $89 \%$ fueron por $P$. vivax y los restantes por $P$. falciparum. El día del ingreso al estudio (día 0), la parasitemia promedio en los casos de malaria por $P$. vivax fue de $9.500 \pm 7.930$ parásitos/ $/ \mu \mathrm{l}$, mientras que, en los casos por $P$. falciparum fue de $11.985 \pm 13.318$ parásitos/ $\mu \mathrm{l}$, sin diferencias estadísticamente significativas entre los grupos. De los niños estudiados, $85 \%$ tenían parásitos intestinales patógenos; $43,5 \%$, Ascaris lumbricoides; $68,2 \%$ Trichuris trichiura, y $37,6 \%$, uncinarias; en las prevalencias de estos parásitos no se presentaron diferencias estadísticamente significativas entre los grupos (cuadro 1).

En la línea de base, los promedios de las variables bioquímicas no presentaron diferencias estadísticamente significativas por grupo de

Cuadro 1. Características de los niños el día de ingreso al estudio, según especie y grupo de tratamiento.

\begin{tabular}{|c|c|c|c|c|c|c|}
\hline \multirow[b]{2}{*}{ Variables } & \multirow[b]{2}{*}{$\begin{array}{l}\text { Total } \\
\mathrm{N}=93\end{array}$} & \multicolumn{4}{|c|}{ Grupo de tratamiento } & \multirow[b]{2}{*}{$\mathbf{p}^{1}$} \\
\hline & & $\begin{array}{l}\text { MAP } \\
n=21\end{array}$ & $\begin{array}{c}\text { MP } \\
n=23\end{array}$ & $\begin{array}{c}\text { MA } \\
n=26\end{array}$ & $\begin{array}{c}M \\
n=23\end{array}$ & \\
\hline Sexo (hombres/mujeres) & $60 / 33$ & $13 / 8$ & $15 / 8$ & $17 / 9$ & $15 / 8$ & $0,944^{1}$ \\
\hline Edad años (media $\pm D E)$ & $7,0 \pm 2,0$ & $6,9 \pm 1,9$ & $7,4 \pm 2,0$ & $7,1 \pm 2,1$ & $6,7 \pm 1,8$ & $0,613^{2}$ \\
\hline Especie, $P$. vivax/P. falciparum & $83 / 10$ & $20 / 1$ & $19 / 4$ & $23 / 3$ & $21 / 2$ & $0,632^{1}$ \\
\hline Peso (kg) (media $\pm D E)$ & $21,6 \pm 5,4$ & $20,7 \pm 5,1$ & $21,5 \pm 5,0$ & $21,4 \pm 5,8$ & $22,7 \pm 5,9$ & $0,742^{2}$ \\
\hline Talla $(\mathrm{cm})$ (media $\pm D E)$ & $117,3 \pm 13$ & $115,9 \pm 12$ & $118,8 \pm 13$ & $117,1 \pm 15$ & $117,4 \pm 13$ & $0,907^{2}$ \\
\hline Parásitos/ul (media $\pm D E)$ & $9.794 \pm 8.678$ & & & & & \\
\hline $10.725 \pm 9.536$ & $8.629 \pm 8.159$ & $9.116 \pm 8.041$ & $10.876 \pm 9.390$ & 0,8082 & & \\
\hline Coprológico $(P / N)^{3}$ & $72 / 13$ & $16 / 4$ & $15 / 5$ & $21 / 2$ & $20 / 2$ & $0,359^{1}$ \\
\hline Ascaris lumbricoides $(\mathrm{P} / \mathrm{N})^{3}$ & $37 / 48$ & $8 / 12$ & $10 / 10$ & $12 / 11$ & $7 / 15$ & $0,499^{1}$ \\
\hline $\begin{array}{l}\text { A. lumbricoides } \mathrm{hpg}^{3} \\
\text { (media } \pm \mathrm{DE})\end{array}$ & $23.288 \pm 51.637$ & $29.000 \pm 5.8971$ & $26.650 \pm 57.853$ & $16.152 \pm 31.485$ & $22.500 \pm 57.917$ & $0,765^{2}$ \\
\hline Trichuris trichiura $(\mathrm{P} / \mathrm{N})^{3}$ & $58 / 27$ & $15 / 5$ & $11 / 9$ & $17 / 6$ & $15 / 7$ & $0,497^{1}$ \\
\hline T. trichiura $(\mathrm{h} / \mathrm{g})(\text { media } \pm \mathrm{DE})^{3}$ & $4.353 \pm 8.475$ & $7.350 \pm 11.611$ & $2.450 \pm 3.154$ & $4.239 \pm 10.068$ & $3.477 \pm 6.107$ & $0,492^{2}$ \\
\hline Uncinarias $(\mathrm{P} / \mathrm{N})^{3}$ & $32 / 53$ & $11 / 9$ & $5 / 15$ & $9 / 14$ & $7 / 15$ & $0,234^{1}$ \\
\hline Uncinarias $(\mathrm{h} / \mathrm{g})(\text { media } \pm \mathrm{DE})^{3}$ & $1.206 \pm 2.993$ & $1.725 \pm 3.358$ & $650 \pm 1.387$ & $1.370 \pm 4.049$ & $1.068 \pm 2.475$ & $0,342^{2}$ \\
\hline
\end{tabular}

MAP: grupo que recibió antipalúdico, retinol y antiparasitario; MP: grupo que recibió antipalúdico y antiparasitario; MA: grupo que recibió antipalúdico y retinol; M; grupo que sólo recibió antipalúdico.

${ }^{1}$ Prueba de ji al cuadrado; ${ }^{2}$ prueba de Kruskall y Wallis; P/N: positivo para parásitos totales/negativo para parásitos totales;

${ }^{3}$ tuvieron coprológico 85 niños: 20 del grupo MAP, 20 del MP, 23 del MA y 22 del M; h/g: número de huevos por gramo de heces y los valores de media $\pm \mathrm{DE}$ se calcularon teniendo en cuenta a todos los sujetos del grupo, positivos y negativos para el parásito. 
tratamiento. A continuación se mencionan sus promedios en el grupo total y el respectivo valor de $p$ de la comparación entre los diferentes grupos; hemoglobina, $10,3 \pm 1,6 \mathrm{~g} / \mathrm{dl}(\mathrm{p}=0,411)$; retinol, $19,1 \pm 6,0 \mu \mathrm{g} / \mathrm{dl}(\mathrm{p}=0,750)$; proteína $C$ reactiva, $75 \pm 63 \mathrm{mg} / \mathrm{l}(\mathrm{p}=0,408)$, y ferritina, $213 \pm 203$ $\mu \mathrm{g} / \mathrm{l}(\mathrm{p}=0,415)$ (no se presentan los datos).

Se calcularon las diferencias entre el valor del día 30 y el de la línea de base (día 0), para la hemoglobina, el retinol, la proteína $\mathrm{C}$ reactiva y la ferritina. Igualmente, se calcularon los cambios parciales, restando el valor de la línea de base al de la segunda medición (día 8) para todas las variables, excepto para la ferritina, resultados que se muestran en el cuadro 2. En la figura 1 se ilustra la evolución en el tiempo de las variables anotadas.

Los cambios de hemoglobina el día 8 fueron pequeños y en el único grupo que aumentó el valor fue en el MAP; de hecho, la fracción de cambio parcial en relación con la línea de base, presentó diferencias estadísticamente significativas entre los grupos (cuadro 2). Entre la segunda y la tercera medición, todos los grupos incrementaron el valor de la hemoglobina (figura 2), de tal forma que el cambio total en relación con la línea de base no presentó diferencias estadísticamente significativas entre los grupos (cuadro 2).
La proteína C reactiva en los cuatro grupos disminuyó dramáticamente a los ocho días de iniciar el tratamiento antipalúdico y las modificaciones posteriores fueron relativamente pequeñas. Sin embargo, el cambio total en relación con la línea de base presentó diferencias estadísticamente significativas en todos los grupos (figura 2). El retinol mostró un comportamiento particular: a los ocho días se incrementó casi al doble del valor de la línea de base en todos los grupos y, posteriormente, tuvo un breve descenso en todos los grupos, excepto en el $\mathrm{M}$, en el cual se incrementó, aunque levemente. No obstante, la fracción de cambio total con referencia a la línea de base dentro de cada grupo, presentó diferencias estadísticamente significativas. La ferritina disminuyó en todos los grupos de tratamiento el día 30, también con diferencias estadísticamente significativas en todos los grupos (figura 2).

Para conocer si las variables presentaban cambio progresivo en el tiempo, en cada grupo se compararon el cambio total y el cambio parcial de cada variable. Las diferencias entre el cambio total y el cambio parcial fueron estadísticamente significativas para la hemoglobina en todos los grupos de tratamiento $(p<0,001)$; lo mismo se encontró para la proteína $C$ reactiva, excepto en el grupo que sólo recibió vitamina A (cuadro 2).

Cuadro 2. Diferencias de los valores de hemoglobina, ferritina, proteína C reactiva y retinol entre la línea de base, la segunda y tercera evaluación, según grupo de tratamiento.

\begin{tabular}{|c|c|c|c|c|c|c|c|c|c|c|c|c|}
\hline \multirow[b]{3}{*}{ Variable } & \multirow[b]{3}{*}{$\begin{array}{l}\text { Periodo } \\
\text { del cambio }\end{array}$} & \multicolumn{11}{|c|}{ Grupo de tratamiento } \\
\hline & & \multicolumn{2}{|c|}{ Total } & \multicolumn{2}{|r|}{ MAP } & \multicolumn{2}{|c|}{ MP } & \multicolumn{2}{|c|}{ MA } & \multicolumn{2}{|r|}{ M } & \multirow[b]{2}{*}{$p^{1}$} \\
\hline & & $n$ & $X \pm D E$ & $\mathbf{n}$ & $X \pm D E$ & $n$ & $X \pm D E$ & n & $X \pm D E$ & $\mathbf{n}$ & $X \pm D E$ & \\
\hline \multirow[t]{2}{*}{ Hemoglobina (mg/dl) } & Día 8 - día 0 & 82 & $-0,1 \pm 1,2$ & 18 & $0,4 \pm 0,8$ & 18 & $-0,2 \pm 1,4$ & 24 & $0,0 \pm 1,2$ & 22 & $-0,7 \pm 1,3$ & 0,020 \\
\hline & $\begin{array}{l}\text { Día } 30 \text { y día } 0 \\
p^{2}\end{array}$ & 77 & $1,4 \pm 1,4$ & 16 & $\begin{array}{c}1,8 \pm 1,1 \\
0,001\end{array}$ & 18 & $\begin{array}{c}1,5 \pm 1,6 \\
0,000\end{array}$ & 22 & $\begin{array}{c}1,2 \pm 1,3 \\
0,001\end{array}$ & 21 & $\begin{array}{c}1,2 \pm 1,6 \\
0,000\end{array}$ & 0,497 \\
\hline \multirow{2}{*}{$\begin{array}{l}\text { Proteína C reactiva } \\
(\mathrm{mg} / \mathrm{L})\end{array}$} & Día 8 - día 0 & 92 & $-68 \pm 64$ & 21 & $-90 \pm 90$ & 22 & $-68 \pm 52$ & 26 & $-52 \pm 62$ & 23 & $-67 \pm 42$ & 0,516 \\
\hline & $\begin{array}{l}\text { Día } 30 \text { y día } 0 \\
\mathrm{p}^{2}\end{array}$ & 83 & $-66 \pm 60$ & 18 & $\begin{array}{l}-96 \pm 95 \\
0,002\end{array}$ & 19 & $\begin{array}{l}-55 \pm 35 \\
0,016\end{array}$ & 24 & $\begin{array}{l}-49 \pm 49 \\
0,989\end{array}$ & 22 & $\begin{array}{c}-70 \pm 42 \\
0,008\end{array}$ & 0,134 \\
\hline \multirow[t]{2}{*}{ Retinol ( $\mu \mathrm{g} / \mathrm{dl})$} & Día 8 - día 0 & 44 & $13,2 \pm 8,6$ & 9 & $12,7 \pm 12,3$ & 12 & 15,2 & 14 & $13 \pm 7,3$ & 9 & $11,4 \pm 8,7$ & 0,670 \\
\hline & $\begin{array}{l}\text { Día } 30 \text { y día } 0 \\
\text { p }^{2}\end{array}$ & 76 & $11,5 \pm 8,1$ & 17 & $\begin{array}{c}10,8 \pm 8,9 \\
0,735\end{array}$ & 19 & $\begin{array}{c}13,2 \pm 7,5 \\
0,424\end{array}$ & 22 & $\begin{array}{l}9,8 \pm 8 \\
0,346\end{array}$ & 18 & $\begin{array}{c}12,3 \pm 8,3 \\
0,327\end{array}$ & 0,308 \\
\hline Ferritina $(\mu g / L)$ & Día 30 y día 0 & 85 & $-184 \pm 203$ & 18 & $-245 \pm 337$ & 21 & $-178 \pm 107$ & 24 & $-161 \pm 188$ & 22 & $-164 \pm 137$ & 0,454 \\
\hline
\end{tabular}

Diferencia día 8-día 0: cambio entre la línea de base y la segunda medición; diferencia día 30-día 0: cambio en la variable, entre la tercera medición y la línea de base; 'valores de $p$ de comparación entre grupos por la prueba de Kruskal y Wallis; ${ }^{2}$ valores de $p$ de la comparación de las dos diferencias por la prueba de Wilcoxon. 
$1 \mathrm{~A})$

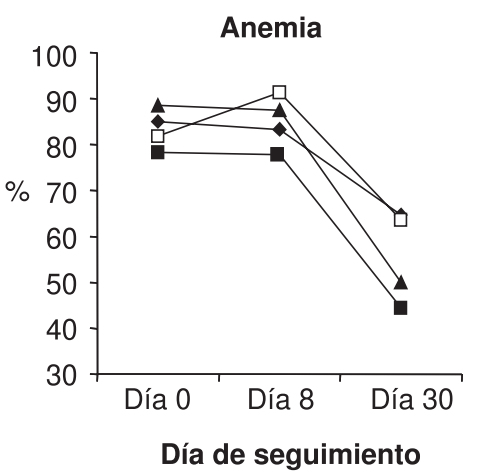

$1 \mathrm{B)}$

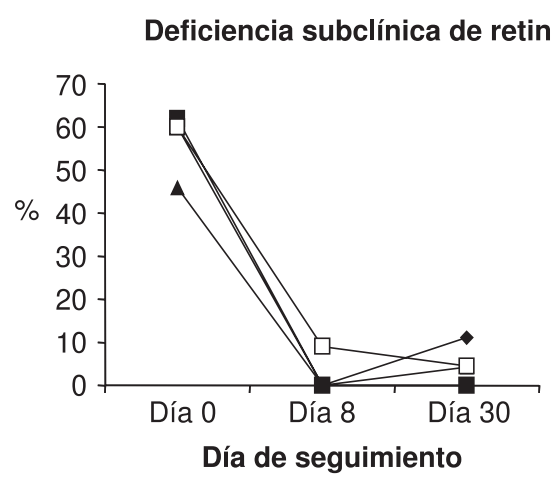

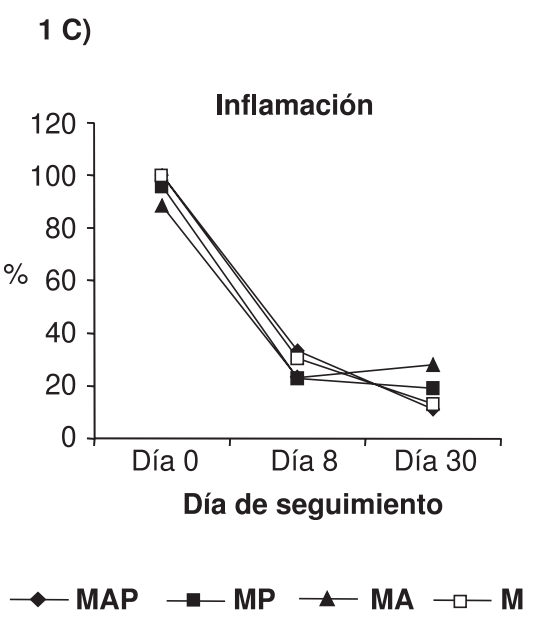

Figura 1. Prevalencia de: $1 \mathrm{~A}$ ) anemia, 1B) deficiencia subclínica de retinol y 1C) inflamación. Las diferencias entre el inicio y el final, según McNemar, fueron para anemia: en grupo MAP, $p=0,375$, y $M, p=0,180$, en los grupos MP y MA, $p<0,02$. Para inflamación, $p<0,000$, en todos los grupos y para deficiencia subclínicas de retinol, $p<0,02$, en todos los grupos. Las diferencias entre grupos según la prueba de ji al cuadrado al inicio y al final fueron: para anemia, $p=0,801, y, p=0,506 ; p a r a$ deficiencia subclínica de retinol, $p=0,669, y, p=0,461$, y para inflamación, $p=0,512, y, p=0,454$.

El retinol no mostró diferencias estadísticamente significativas entre el cambio parcial y el cambio total, en ninguno de los grupos. Cabe anotar que para la ferritina no se pudo comparar la diferencia entre los cambios parcial y total, porque no se midió el día 8. Con excepción del cambio parcial de la hemoglobina, que mostró diferencias estadísticamente significativas entre los grupos de tratamiento, no se observaron estas diferencias, ni en los cambios parciales ni en los totales, en las demás variables estudiadas (cuadro 2).
Los cambios en los porcentajes de anemia, entre la línea de base y el final del estudio, fueron de 18 unidades en el grupo M, 20 en el MAP, 24 en el MP y, finalmente, 38 en el MA; estos cambios únicamente fueron estadísticamente significativos en los dos últimos grupos mencionados (figura 1).

Las frecuencias de deficiencia subclínica de retinol y de inflamación no mostraron diferencias estadísticamente significativas entre los grupos, ni en la línea de base ni al final del estudio, pero, los cambios en cada grupo sí presentaron esas 
$1 \mathrm{~A})$

Hemoglobina

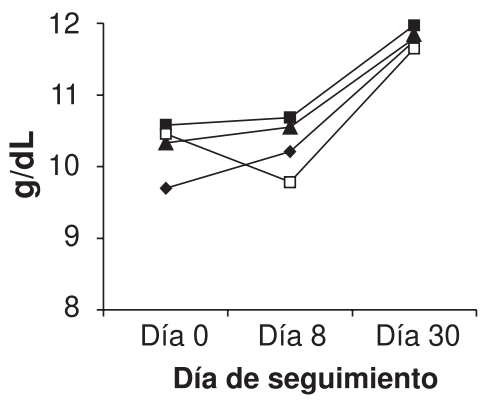

1C)

Retinol

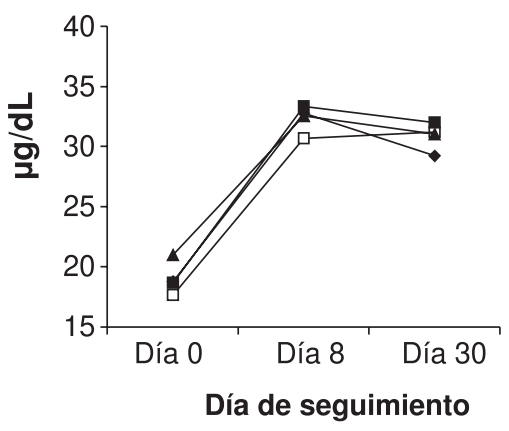

$1 \mathrm{B)}$

Proteína $\mathrm{C}$ reactiva

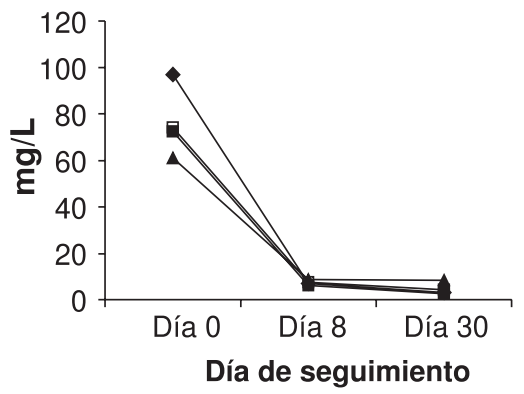

1D)

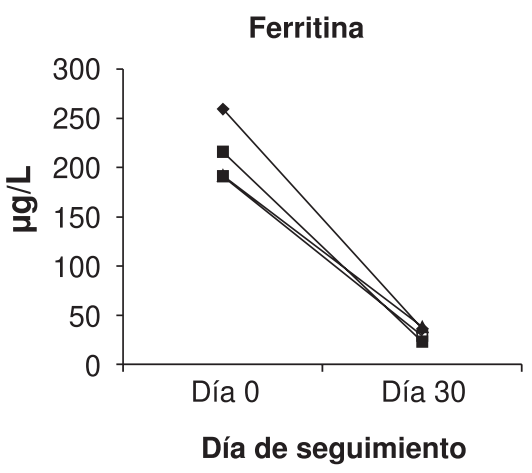

Grupos: $\multimap$ MAP $\rightarrow$ MP $\multimap$ MA $\square-M$

Figura 2. Concentraciones de: $1 \mathrm{~A}$ ) hemoglobina, 1B) proteína $\mathrm{C}$ reactiva, $1 \mathrm{C}$ ) retinol y $1 \mathrm{D}$ ) ferritina según grupo de tratamiento y día de seguimiento.

MAP: grupo de antipalúdico, retinol y antiparasitario; MP: grupo de antipalúdico y antiparasitario; MA: grupo de antipalúdico y retinol; M: grupo de antipalúdico. La diferencia entre la medición final (día 30) y la línea de base (día 0) para todas las variables de los diferentes grupos, mostró valores de $p<0,002$, según la prueba de Wilcoxon.

diferencias entre el inicio y el final del estudio (figura 1). En el total de sujetos, la inflamación se redujo de $96 \%$ a $18 \%$ y, la deficiencia subclínica de retinol, de $56 \%$ a $5 \%$ (figura 1 ).

\section{Discusión}

Las concentraciones de retinol en nuestro estudio fueron más bajas que las informadas previamente en niños palúdicos del municipio de El Bagre (25). Estos hallazgos concuerdan con los reportes de niños con malaria por $P$. falciparum, en los que se encontraron bajas concentraciones de retinol $(26,27)$.
Los cambios en el retinol sérico no fueron progresivos en el tiempo y ocurrieron en mayor magnitud durante los primeros ocho días. Sin embargo, el retinol del día 30, después de haber recibido el tratamiento antipalúdico, fue significativamente mayor que en la línea de base en todos los grupos de tratamiento y el cambio total fue independiente de haber recibido suplemento de vitamina A o antiparasitarios o de no haberlo recibido. No obstante, un elemento que dificulta el análisis de los cambios de retinol el día 8 fue el número reducido de niños que contaron con esta determinación. 
Tampoco se observó diferencia entre los grupos en la prevalencia de la deficiencia subclínica de vitamina $A$ al final del estudio, que fue de $5 \%$ en el total, cifra similar a la prevalencia nacional en los niños colombianos de 1 a 4 años informada en la última Encuesta Nacional de Salud y Nutrición, 2005 (28); de esto se deduce que el factor predominante que influyó sobre el retinol plasmático fue la respuesta inflamatoria, de acuerdo con estudios previos donde se reportó reducción de las concentraciones de retinol durante la fase aguda de diversas infecciones (29), las cuales se incrementan una vez curada la infección, como se informó previamente en niños palúdicos colombianos (25).

La falta de efecto del suplemento de retinol y de los antiparasitarios intestinales sobre los cambios en el retinol plasmático en los niños del estudio que estaban altamente parasitados, fue contrario a lo que se ha observado en niños no palúdicos, en quienes los suplementos de retinol aumentaron las concentraciones séricas del mismo y redujeron la prevalencia de deficiencia subclínica de retinol $(30,31)$, lo mismo que la administración de antihelmínticos (32). Las diferencias podrían deberse a que la prevalencia de la deficiencia subclínica de retinol es baja en los niños colombianos habitantes de zonas palúdicas $y$, en consecuencia, los valores de retinol no mejoran con los suplementos ni con la administración de antihelmínticos. No obstante, hay que tener en cuenta que las concentraciones de retinol aumentan con la edad, el índice de masa corporal y los lípidos séricos, y están relacionadas con la inflamación (33) y el proceso de maduración puberal (34), al mismo tiempo que están sujetas a regulación homeostática y no disminuyen hasta que las reservas hepáticas estén peligrosamente bajas. En conclusión, se afectan por gran cantidad de factores y, por lo tanto, no son buenos indicadores de las reservas orgánicas de vitamina A (35).

El cambio total en la hemoglobina fue significativamente mayor que el parcial en todos los grupos de tratamiento, sin diferencias por grupo en cuanto al cambio total, pero sí en el cambio entre la línea de base y la segunda medición (día 8), en el cual la administración conjunta de un suplemento de retinol y antiparasitarios intestinales al inicio del tratamiento antipalúdico evitó que la hemoglobina disminuyera; incluso, fue el único grupo en que aumentó $(0,4 \pm 0,8 \mathrm{~g} / \mathrm{dl})$ y se previno el incremento de la anemia.

No obstante, ese efecto no se vio reflejado al final del estudio ni en las concentraciones de hemoglobina, ni en las prevalencias de anemia. Puesto que esta investigación se realizó en niños con malaria no complicada, entre quienes es poco frecuente encontrar anemia grave, no fue posible probar si la intervención con antiparasitarios y suplemento de retinol podría evitar la aparición de este tipo de anemia asociada con alta mortalidad entre los niños con malaria complicada (36). Dado el reducido número de niños con malaria por $P$. falciparum, tampoco fue posible evaluar si el efecto de la intervención en mención difiere según la especie de parásito que ocasiona la malaria. El aumento de las concentraciones de hemoglobina en el día 30 podría ser el resultado de la reducción de la hemólisis una vez tratada la malaria y, el posterior incremento en la eritropoyesis, en una respuesta a la hipoxia tisular asociada con la anemia. Las altas demandas de hierro para producir glóbulos rojos a mayor velocidad, podrían haberse obtenido de los depósitos de ferritina, puesto que el hierro tiene un circuito cerrado en los humanos (37).

Los porcentajes de anemia durante la malaria aguda fueron muy superiores a los encontrados por Blair et al. en niños de El Bagre (1997) (11), pero inferiores a los reportados en niños venezolanos $(95 \%)$ infectados con $P$. vivax (38). En el día 30, la prevalencia de anemia (56\%) fue muy superior a la informada en la Encuesta Nacional de Salud y Nutrición, 2005, para los niños de 5 a 12 años habitantes de la región central (33\%), donde se encuentra Antioquia y, por supuesto, los municipios de El Bagre y Turbo (28). Estos hallazgos están de acuerdo con los de otros autores, quienes afirman que la malaria aumenta 4,1 veces el riesgo de desarrollar anemia (39). La proporción de sujetos anémicos al final del estudio puede reflejar en parte el estado hematológico previo al paludismo. Sin embargo, puesto que el estudio 
finalizó el día 30 y no se observaron los cambios posteriores en las variables estudiadas, no se sabe si posteriormente la hemoglobina continuó aumentando y la anemia disminuyendo.

La administración de un suplemento de retinol y de antiparasitarios intestinales, solos o combinados, tampoco afectó los cambios en las concentraciones de proteína $\mathrm{C}$ reactiva y ferritina, ni a los 8 ni a los 30 días después de su administración en niños con malaria no complicada. Las concentraciones de ferritina al inicio del estudio fueron muy altas, lo que se explica porque esta proteína se comporta como un reactante positivo de fase aguda (8) y es posible que la hemólisis asociada con la malaria haya contribuido a incrementarlas. Se sabe que, durante un episodio agudo de paludismo, se incrementa la fagocitosis por los macrófagos de los glóbulos rojos parasitados, al mismo tiempo que otros no parasitados se tornan frágiles y se produce hemólisis intravascular (40). Igualmente, es conocido que en los humanos el hierro tiene un circuito cerrado y el proveniente de la hemólisis de los glóbulos rojos se deposita como ferritina (37).

La respuesta inflamatoria durante la malaria, evaluada mediante las concentraciones de proteína $\mathrm{C}$ reactiva, fue muy fuerte y se redujo dramáticamente una vez administrado el tratamiento antipalúdico, pero no se observaron diferencias atribuibles a la intervención con un suplemento de retinol y antiparasitarios intestinales. Este comportamiento podría deberse a que el efecto de los antipalúdicos sobre la respuesta inflamatoria es muy potente y se superpone con el posible efecto que haya tenido la intervención. De hecho, la variabilidad de los datos de proteína $C$ reactiva es muy alta, lo cual sugiere, además, que hay otras variables que pueden estar influyendo sobre ella. Para aclarar este aspecto, se requiere de otro estudio con una muestra más grande, donde se pueda explorar el comportamiento de la proteína $C$ reactiva en niños palúdicos, estratificando por edad y estado nutricional.

Un factor que pudo influenciar los resultados y que se refleja en la gran variabilidad de los datos, fue que no se completó la muestra prevista inicialmente de 104 niños por la dificultad para garantizar la asistencia de los padres o acudientes de los niños que cumplían los requisitos de admisión, a las consultas de seguimiento en tres ocasiones, porque la mayoría de ellos procedían de zonas rurales alejadas y de difícil acceso. A esto se sumó que, al finalizar el estudio, algunos niños no permitieron que se les tomara la muestra de sangre. Además, el número de participantes del día 30 se redujo aún más cuando se eliminaron del análisis tres niños que presentaron gota gruesa positiva. Sólo se incluyeron los niños que pudieron ser captados durante un año, dados los altos costos de sostenimiento de dos equipos de trabajo de campo.

En conclusión, el suplemento de retinol y la desparasitación intestinal simultánea con el tratamiento antipalúdico, no influyeron sobre los cambiostotalesy parciales enlasconcentraciones de retinol, ferritina y proteína $C$ reactiva en niños con malaria aguda, pero sí evitaron que el día 8 las concentraciones de hemoglobina continuaran disminuyendo y las de anemia aumentando, aunque estos cambios iniciales no mostraron efecto un mes después. Se requiere de estudios con tamaños de muestra más grandes, para medir el resultado de estas intervenciones, y otros que permitan entender cómo los suplementos de retinol y los antiparasitarios intestinales administrados simultáneamente, modulan el metabolismo del hierro, antes de recomendar su uso como parte del tratamiento antipalúdico.

\section{Agradecimientos}

A las comunidades de El Bagre y de Turbo, a los directivos y personal de los hospitales de Turbo y El Bagre y sus puestos de malaria; igualmente, a los profesionales Alexandra Ríos, Juan Gabriel Piñeros, Tania Álvarez, José Nicolás Martínez y Patricia Rocha, quienes nos apoyaron en el trabajo de campo. A los epidemiólogos Alejandro Estrada y Bertha Lucía Gaviria, por su asesoría estadística.

\section{Conflicto de intereses}

Los autores manifiestan que no existe ningún conflicto de interés. 


\section{Financiación}

El estudio fue financiado por el Comité para el Desarrollo de la Investigación de la Universidad de Antioquia (CODI), el Instituto Colombiano para el Desarrollo de la Ciencia y la Tecnología (Colciencias), código 1115-04-16388, contrato 339-2004 y la Dirección Seccional de Salud de Antioquia (DSSA), convenio administrativo $\mathrm{Cl}$ 021-2004.

\section{Referencias}

1. Dirección Seccional de Salud de Antioquia. Eventos de interés en salud pública, Antioquia 2006. Fecha de consulta:20 de mayo de 2008. www.dssa.gov.co/dowload/ archivoseventos_2007/EnotificacionSPVer2006-2.xls

2. Castro L, Nicholls S. Deficiencia de hierro, vitamina A y prevalencia de parasitismo intestinal en población infantil y anemias nutricionales en mujeres en edad fértil de Colombia en 1995-1996. Santafé de Bogotá: Instituto Nacional de Salud; 1998. p. 50-5.

3. Ordóñez LE, Angulo ES. Desnutrición y su relación con parasitismo intestinal en niños de una población de la Amazonia colombiana. Biomédica. 2002;22:486-98.

4. Carmona-Fonseca J. La malaria en Colombia, Antioquia y las zonas de Urabá y Bajo Cauca: panorama para interpretar la respuesta terapéutica antimalárica. Parte 1. latreia. 2003;16:299-318.

5. Carmona-Fonseca J. La malaria en Colombia, Antioquia y las zonas de Urabá y Bajo Cauca: panorama para interpretar la respuesta terapéutica antimalárica. Parte 2. Iatreia. 2004;17:354-69.

6. van den Berg $\mathbf{H}$. Vitamin A intake and status. Eur J Clin Nutr. 1996;50(Suppl.3):7-12.

7. WHO/NHD. Iron deficiency anaemia, assessment, prevention, and control a guide for programme managers. Geneva: United Nations Children's Fund, United Nations University, World Health Organization; 2001. p. 10.

8. TomkinsA.Assessing micronutrientstatusinthe presence of inflammation. J Nutr. 2003;133(Suppl.2):1649-55.

9. Hurt N, Smith T, Teuscher T, Tanner M. Do high levels of C-reactive protein in Tanzanian children indicate malaria morbidity? Clin Diagn Lab Immunol. 1994; 1:437-44.

10. Blair S, Álvarez G, Villa A, Carmona-Fonseca J, Ríos L. Estado nutricional y niveles de inmunoglobulinas y citoquinas en niños con malaria. Anales de Pediatría. 2003;58:418-24.

11. Blair S, Álvarez G, Campuzano G. Relación entre anemia y malaria en una población rural colombiana. Boletín de la Dirección de Malariología y Saneamiento Ambiental. 1997;37:7-12.
12. Echeverri M, Tobón A, Álvarez G, Carmona J, Blair S. Clinical and laboratory findings of Plasmodium vivax malaria in Colombia, 2001. Rev Inst Med Trop Sao Paulo. 2003;45:29-34

13. Persson V, Ahmed F, Gebre-Medhin M, Dreiner T. Relationships between vitamin $A$, iron status and helminthiasis in Bangladeshi school children. Public Health Nutr. 2000;3:83-9.

14. Koski KG, Scott ME. Gastrointestinal nematodes, nutrition and immunity: breaking the negative spiral. Annu Rev Nutr. 2001;21:297-321.

15. World Health Organization. Severe falciparum malaria. Severe and complicated malaria. Trans R Soc Trop Med Hyg. 2000;94(Suppl.1):1-88.

16. Willows ND, Gray-Donal K. Serum retinol is associated with hemoglobin concentration in infants who are not vitamin A deficient. Nutr Res. 2003;23:891-900.

17. Stoltzfus RJ, Albonico M, Chwaya HM, Tielsch JM, Schulze KJ, Savioli L. Effects of the Zanzibar schoolbased deworming program on iron status of children. Am J Clin Nutr. 1998;68:179-86.

18. Stoltzfus RJ, Chway HM, Montresor A, Tielsch JM, Jape JK, Albonico M, et al. Low dose daily iron supplementation improves iron status and appetite but not anemia, whereas quarterly antihelminthic treatment improves growth, appetite and anemia in Zanzibari preschool children. J Nutr. 2004;134:348-56.

19. Mwanri L, Worsley A, Ryan P, Masika J. Supplemental vitamin A improves anemia and growth in anemic school children in Tanzania. J Nutr. 2000;130:2691-6.

20. López F, Schmunis G. Diagnóstico de malaria. Publicación científica 512. Washington, D.C.: OPS-OMS; 1988. p. 143.

21. Rice CP, Trull AK, Berry D, Gorman EG. Development and validation of a particle-enhanced turbidimetric immunoassay for C-reactive protein. J Immunol Methods. 1987;99:205-11.

22. Talwar D, Ha TK, Cooney J, Brownlee C, O’Reilly DS. A routine method for the simultaneous measurement of retinol, alpha-tocopherol and five carotenoids in human plasma by reverse phase HPLC. Clin Chim Acta. 1998;270:85-100.

23. Uscátegui RM, Correa AM. Estado nutricional de niños palúdicos residentes en El Bagre y Turbo, Antioquia, Colombia, 2004-2005. Biomédica. 2007;27:559-70.

24. Botero D, Restrepo M. Parasitosis humanas. Cuarta edición. Medellín: Corporación para Investigaciones Biológicas; 2003. p. 455-63.

25. Blair S, Carmona J, Correa AM. Malaria en niños: relaciones entre nutrición e inmunidad. Rev Panam Salud Pública. 2002;11:5-14.

26. Rosales FJ, Topping JD, Smith JE, Shankar AH, Ross AC. Relation of serum retinol to acute phase proteins 
and malarial morbidity in Papua, New Guinea, children. Am J Clin Nutr. 2000;71:1582-8.

27. Filteau SM, Willumsen JF, Sullivan K, Simmank K, Gamble M. Use of the retinol-binding protein: transthyretin ratio for assessment of vitamin A status during the acutephase response. Br J Nutr. 2000;83:513-20.

28. Álvarez MC, Correa JM, Deossa GC, Estrada A, Forero Y, Gómez LF et al. Encuesta Nacional de la Situación Nutricional en Colombia. Bogotá: ICBF, Profamilia, Instituto Nacional de Salud, Universidad de Antioquia, OPS, Panamericana Formas e Impresos; 2005. p. 445.

29. Thurnham DI, McCabe GP, Northrop-Clewes CA, Nestel P. Effects of subclinical infection on plasma retinol concentrations and assessment of prevalence of vitamin A deficiency: meta-analysis. Lancet. 2003;362:2052-8.

30. Rahman MM, Wahed MA, Fuchs GJ, Baqui AH, Álvarez JO. Synergistic effect of zinc and vitamin A on the biochemical indexes of vitamin A nutrition in children. Am J Clin Nutr. 2002;75:92-8.

31. Zimmermann MB, Biebinger R, Rohner F, Dib A, Zeder C, Hurrell RF, et al. Vitamin A supplementation in children with poor vitamin $A$ and iron status increases erythropoietin and hemoglobin concentrations without changing total body iron. Am J Clin Nutr. 2006;84:580-6.

32. Tanumihardjo SA, Permaesih D, Muhilal. Vitamin A status and hemoglobin concentrations are improved in Indonesian children with vitamin A and deworming interventions. Eur J Clin Nutr. 2004;58:1223-30.

33. Stephensen CB, Gildengorin G. Serum retinol, the acute phase response, and the apparent misclassification of vitamin A status in the third National Health and Nutrition Examination Survey. Am J Clin Nutr. 2000;72:1170-8.

34. Álvarez MC, Uscátegui RM, López C, Baracaldo CM, Castro L, Noy V. Plasma retinol concentration according to pubertal maturation in school children and adolescents of Medellin, Colombia. Eur J Clin Nutr. 2004;58:456-61.

35. Tanumihardjo SA. Assessing vitamin A status: past, present and future. J Nutr. 2004;134(Suppl.1):290-3.

36. Obonyo CO, Vulule J, Akhwale WS, Grobbee DE. In-hospital morbidity and mortality due to severe malarial anemia in western Kenya. Am J Trop Med Hyg. 2007;77:23-8

37. Anderson GJ, Darshan D, Wilkins SJ, Frazer DM. Regulation of systemic iron homeostasis: how the body responds to changes in iron demand. Biometals. 2007;20:665-74.

38. Rodríguez-Morales AJ, Sánchez E, Vargas M, Piccolo C, Colina R, Arria M. Anemia and thrombocytopenia in children with Plasmodium vivax malaria. J Trop Pediatr. 2006;52:49-51.

39. Villamor E, Mbise R, Spiegelman D, Ndossi G, Fawzi WW. Vitamin A supplementation and other predictors of anemia among children from Dar Es Salaam, Tanzania. Am J Trop Med Hyg. 2000;62:590-7.

40. Roberts DJ, Casals-Pascual C, Weatherall DJ. The clinical and pathophysiological features of malaria anaemia. En: Sullivan J, Krishna S, editors. Malaria: drugs, disease and post-genomic biology. Berlin: Springer-Verlag Berlin Heidelberg; 2005. p. 137-8. 